\title{
Epidemiological and Microbiological Aspects of Acute Bacterial Diarrhea in Children from Salvador, Bahia, Brazil
}

\author{
Daniel R. Diniz-Santos ${ }^{1}$, José S. Santana ${ }^{1}$, \\ Junaura R. Barretto ${ }^{1}$, Maria Goreth M. Andrade ${ }^{2}$ \\ and Luciana R. Silva ${ }^{1}$
}

\author{
Pediatric Gastroenterology and Hepatology Division ${ }^{1}$, \\ Professor Hosannah Oliveira Pediatric Center, School of \\ Medicine, Federal University of Bahia; Laboratory of \\ Microbiology², Hospital Aliança, Salvador, Bahia, Brazil
}

\begin{abstract}
In the few cases of acute childhood diarrhea that require antimicrobial therapy, the correct choice of the drug depends on detailed previous knowledge of local strains. In order to establish such parameters in our city, we reviewed the results of all 260 positive stool cultures of children between 0 and 15 years of age during two years at a pediatric tertiary care facility in Salvador, Brazil. Bacterial strains had been presumptively identified by culturing in selective media and by biochemical testing, and their antimicrobial susceptibility patterns were automatically detected by the MicroScan Walkaway System. Data about patients' sex and age, monthly distribution of the cases, pathogens isolated and their antimicrobial resistance patterns were recorded. Males corresponded to $55.4 \%$ of our sample, and most of our patients $(42.7 \%)$ were between one and four years of age. Shigella was the commonest pathogen, being found in $141(54.3 \%)$ cultures, while Salmonella was found in 100 (38.4\%) cultures and Enteropathogenic E. coli in 19 (7.3\%). Salmonella was the main causal agent of diarrhea in children younger than five years old, whereas Shigella was the most frequent pathogen isolated from the stools of children between five and 15 years old. The peaks of incidence correspond to the periods of school vacations. Shigella specimens presented a very high resistance rate to trimethoprim-sulfamethoxazole $(90.1 \%)$ and to ampicillin

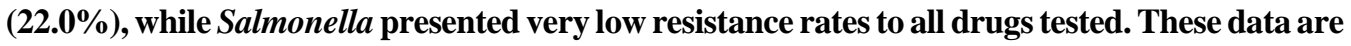
useful for practitioners and they reinforce the need for continuous microbiological surveillance. Key Words: Diarrhea, antimicrobial, resistance, children, epidemiology.
\end{abstract}

Severe presentations of acute diarrhea constitute one of the commonest challenges faced by the medical team in pediatric ambulatories and emergency rooms in the developing world. Supportive anti-dehydration treatment is the cornerstone of therapy and must be promptly started, but specific antimicrobial treatment may be required, depending on the severity of the disease and on the risk of complications. As stool

Received on 19 September 2004; revised 12 January 2005.

Address for correspondence: Dr. Daniel R. Diniz-Santos. Av. Princesa Isabel, 549, apt. 11, Barra Avenida, Salvador, Bahia, Brazil. Zip code: 40130-030. Phone/Fax: (55) (71) 264-4424. Email:danieldiniz82@ig.com.br

The Brazilian Journal of Infectious Diseases 2005;9(1):77-83 (C) 2005 by The Brazilian Journal of Infectious Diseases and Contexto Publishing. All rights reserved. cultures take several days to provide adequate information about pathogens and their susceptibility patterns, empirical treatment must be immediately adopted in such cases. To guide the empirical choice of antibiotics, it is crucial to know both which pathogens are most likely to be infecting the patient in a particular geographic area and the most effective antibiotics for treating them.

All over the world, severe acute bacterial gastroenteritis is caused mainly by Shigella, whereas Salmonella, E. coli (chiefly enteropathogenic E. coli, or EPEC, but also enterohemorrhagic E. coli or EHEC, enteroinvasive E. coli or EIEC and other types), Campylobacter and Vibrio spp. have also been shown to play a role in the epidemiology of diarrhea, especially in certain areas of the globe [1-5]. 
Some years ago, the World Health Organization (WHO) suggested that bloody diarrhea in children should be treated with trimethoprim-sulfamethoxazole (TMP-SMX) or nalidixic acid. However, antimicrobial susceptibility patterns are ever-changing and are highly variable from one geographic zone to another. Cases of bloody acute bacterial gastroenteritis have been successfully treated with ampicillin or TMP-SMX for a long time, but reports of growing resistance have limited the empirical use of those drugs, even though they may have a role in therapy guided by the results of stool cultures. Nalidixic acid initially replaced ampicillin and TMP-SMX satisfactorily, but as resistance emerged and spread quickly [6] it has no longer been considered a mainstay of antimicrobial therapy of bacterial diarrhea. Currently, third generation cephalosporins and the newer quinolones, chiefly ceftriaxone and ciprofloxacin, respectively, are considered the most effective drugs for the selected cases of acute infectious diarrhea in which antimicrobial treatment is indicated. In spite of the profusion of reports from different parts of the world concerning trends in antimicrobial resistance, few data addressing such patterns in Brazil are available, especially for the pediatric population.

This study was carried out to identify and to establish the antimicrobial susceptibility pattern of the most important pathogens involved in the epidemiology of acute diarrhea in children who sought medical attendance at a pediatric tertiary care facility in Salvador, Brazil. General demographic data about the patients and the disease were also recorded.

\section{Material and Methods}

\section{$\underline{\text { Patients }}$}

We reviewed the results of all stool cultures that presented bacterial growth that were taken from children between 0 and 15 years of age from January 2002 to December 2003 at a pediatric tertiary care facility in Salvador, Brazil. Data about patients's sex and age, monthly distribution of the cases, pathogens isolated and their antimicrobial resistance patterns were recorded. During that period, 1,991 patients had their stools cultured and 260 samples presented bacterial growth.

\section{Bacteriology}

All bacteriological examinations were performed at the Hospital Aliança microbiology laboratory. Briefly, fresh feces were collected from diarrhoeal patients and sent to the laboratory for immediate culture. The specimens were inoculated into selenite broth and on MacConkey, Salmonella-Shigella (SS) and Karmali agars.

MacConkey and SS agar plates were incubated at $35^{\circ} \mathrm{C}$ for 18 to 24 hours, and selenite broth at $35^{\circ} \mathrm{C}$ for 12-18 hours. After incubation, selenite broth was inoculated in SS agar at $35^{\circ} \mathrm{C}$ for 18 to 24 hours. Suspected colonies were inoculated in EPM-MILICitrate medium at $35^{\circ} \mathrm{C}$ for 18 to 24 hours for biochemical testing and presumptive identification. After biochemical testing, colonies were tested for serum agglutination with polyvalent sera (Probac) against Shigella sonnei, Shigella flexneri, Shigella boydii and Shigella dysenteriae, classic enteropathogenic $E$. coli A, B and C, entereoinvasive E. coli A and B, and flagellar and somatic Salmonella antigens.

Karmali agar plates were incubated under microaerophilia at $42^{\circ} \mathrm{C}$. After 48 hours, any colonies found were assessed for Campylobacter spp. by Gram staining.

Shigella, Salmonella and E. coli colonies identified on serum agglutination had their identification confirmed and their antimicrobial susceptibility patterns established by microdilution in a WalkAway-96 machine (Dade Behring, West Sacramento, California, USA). Standardization and quality control tests were performed every week using E. coli ATCC25922, as currently recommended by the National Committee for Clinical Laboratory Standards (NCCLS) [7]. We used the Neg BP Combo 12 panel (B1017-131, Dade Behring, West Sacramento, California, USA). The antibiotics tested for susceptibility and their respective concentrations were as follows: ampicillin $(8 \mu \mathrm{g} / \mathrm{mL}$ and 
$16 \mu \mathrm{g} / \mathrm{mL})$, ampicillin + sulbactam $(8 / 4 \mu \mathrm{g} / \mathrm{mL}$ and $16 /$ $8 \mu \mathrm{g} / \mathrm{mL}$ ), aztreonan (8 $\mu \mathrm{g} / \mathrm{mL}$ and $16 \mu \mathrm{g} / \mathrm{mL})$, ceftazidime $(8 \mu \mathrm{g} / \mathrm{mL}$ and $16 \mu \mathrm{g} / \mathrm{mL})$, ceftriaxone (8 $\mu \mathrm{g} / \mathrm{mL}$ and $32 \mu \mathrm{g} / \mathrm{mL})$, ciprofloxacin $(1 \mu \mathrm{g} / \mathrm{mL}$ and 2 $\mu \mathrm{g} / \mathrm{mL})$, imipenem $(4 \mu \mathrm{g} / \mathrm{mL}$ and $8 \mu \mathrm{g} / \mathrm{mL})$, piperacillin (16 $\mu \mathrm{g} / \mathrm{mL}$ and $64 \mu \mathrm{g} / \mathrm{mL})$, and trimethoprim + sulfamethoxazole $(2 / 38 \mu \mathrm{g} / \mathrm{mL})$. Strains were evaluated for extended spectrum beta-lactamase (ESBL)production by the MicroScan System and those described as possible ESBL-producers were further tested by the double disk synergy test in Mueller-Hinton agar, as recommended by NCCLS [7].

\section{Results}

During the two-year period, the stools of 1,991 diarrhoeal patients aged 0 to 15 years were cultured and 260 (13.1\%) samples presented bacterial growth and were included in the study. Of these patients, 144 (55.4\%) were male, while the other 116 (44.6\%) were females. Only nine (3.5\%) of them were younger than one year, while 111 (42.7\%) were between one and four years of age, 81 (31.1\%) were between five and nine years and 59 (22.7\%) between 10 and 15 years (Figure 1).

Shigella spp. was the most frequent pathogen, being found in 141 (54.3\%) cultures (113, or $80.1 \%$, were S. sonnei and 28 , or $19.9 \%$, were S. flexneri), while Salmonella spp. was found in 100 (38.4\%) cultures and E. coli was found in 19 (7.3\%). No typhoidal Salmonella spp. specimens were isolated. The age distribution data revealed that Salmonella was the main causal agent of diarrhea, both in children younger than one year and in those between one and four years-old, whereas Shigella was the most frequent pathogen isolated from the stools of children between five and nine years-old and between 10 and 15 years old (Table 1). E. coli was an uncommon cause of diarrhea in our sample, occurring mostly in children between one and four years old.

We observed a peak of incidence in the summer: 20 cases $(7.7 \%)$ in January, 33 (12.7\%) in February and 24 (9.2\%) in March and another in the winter, in
June (36 cases, or 13.8\%) and July (29 cases, or $11.1 \%)$. Shigella spp. was the pathogen with the highest impact on the epidemiology of acute bacterial diarrhea in these children, since it was responsible for most cases during most months of the year, especially during the peak months of incidence (Table 2).

Considering all 260 pathogens isolated, 135 (51.9\%) were found to be resistant to TMP-SMX and $40(15.4 \%)$ to ampicillin, while 22 (8.5\%) were resistant to ampicillin + sulbactam and 18 (6.9\%) to piperacillin. Low resistance rates were found to ciprofloxacin among Shigella (0.7\%) and Salmonella (2.0\%) isolates. None of the 260 isolates was found to be resistant to ceftriaxone or to aztreonan, and no ESBL-producing strain was isolated.

In a separate assessment of antimicrobial resistance patterns, Shigella specimens presented a very high resistance rate to TMP-SMX (90.1\%). Among $S$. sonnei isolates, TMP-SMX resistance rates were as high as $94.7 \%$, while it reached $71.4 \%$ among $S$. flexneri isolates. These Shigella isolates also presented significant resistance rates to ampicillin, either alone (22.0\%) or in combination with sulbactam (9.9\%). This was especially true for $S$. flexneri isolates, which presented resistance rates as high as 60.8\% (ampicillin alone) and $14.3 \%$ (ampicillin + sulbactam) (Table 3). Otherwise, Salmonella isolates presented very low resistance rates, varying from 1 to $5 \%$, to all the drugs, including TMP-SMX (2.0\%). E. coli isolates presented high resistance rates to ampicillin (both alone and associated with sulbactam), piperacillin and TMP-SMX.

\section{Discussion}

Given the importance of a careful characterization of the local epidemiology of diarrhea to guide specific antimicrobial therapy, we reviewed the results of 260 stool cultures from children aged 0 to 15 years old in which enteropathogens were detected. We detected a discretely higher propensity of males to suffer from bacterial gastroenteritis and a low incidence during the first year of life, a peak of incidence between one and 
Figure 1. Distribution of patients with diarrhea according to age range

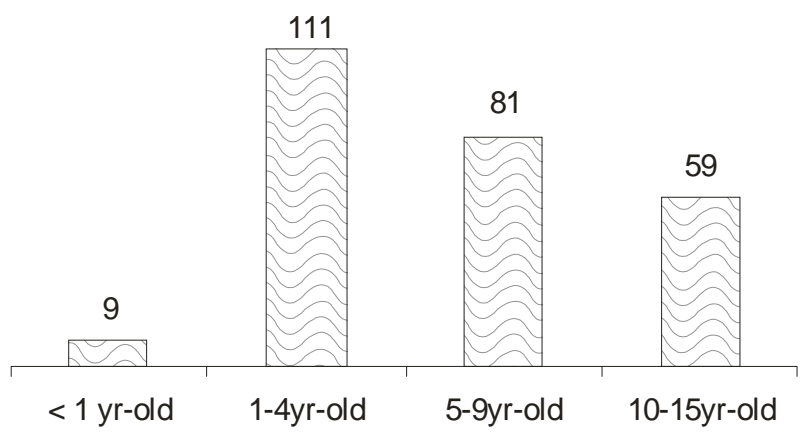

Table 1. Distribution of the pathogens in diarrheal stools according to patients' age

\begin{tabular}{lccrr}
\hline & Shigella spp. & Salmonella spp. & E. coli & \multicolumn{1}{c}{ Total } \\
\hline$<1$ yr-old & 0 & $7(77.8 \%)$ & $2(22.2 \%)$ & $9(100 \%)$ \\
$1-4$ yr-old & $33(29.7 \%)$ & $61(54.6 \%)$ & $17(15.7 \%)$ & $111(100 \%)$ \\
$5-9$ yr-old & $61(75.3 \%)$ & $20(24.7 \%)$ & 0 & $81(100 \%)$ \\
$10-15$ yr-old & $47(79.7 \%)$ & $12(20.3 \%)$ & 0 & $59(100 \%)$ \\
Total & 141 & 100 & 19 & 260 \\
\hline
\end{tabular}

Table 2. Monthly distribution of acute bacterial gastroenteritis according to the pathogenic agent

\begin{tabular}{lrrrrrrrrrrrr}
\hline & Jan & Feb & Mar & Apr & May & Jun & Jul & Aug & Sep & Oct & Nov & Dec \\
\hline Shigella spp. & 6 & 20 & 13 & 6 & 8 & 24 & 22 & 11 & 9 & 11 & 7 & 4 \\
Salmonella spp. & 11 & 13 & 9 & 9 & 7 & 9 & 6 & 3 & 10 & 3 & 14 & 7 \\
E. coli & 3 & 0 & 2 & 2 & 0 & 3 & 1 & 2 & 1 & 0 & 3 & 1 \\
\hline Total & 20 & 33 & 24 & 17 & 15 & 36 & 29 & 16 & 20 & 14 & 24 & 12 \\
$\%$ & 7.7 & 12.7 & 9.2 & 6.5 & 5.8 & 13.8 & 11.1 & 6.2 & 7.7 & 5.4 & 9.2 & 4.6 \\
\hline
\end{tabular}

four years of age (the period when children's contact with environmental pathogens increases dramatically) and decreasing incidence levels in older children (Figure $1)$.

More than half of the cases were caused by Shigella spp., reinforcing the importance of this pathogen in the epidemiology of bacterial childhood diarrhea in developing countries. Most of the Shigellaassociated cases $(80.1 \%)$ were due to $S$. sonnei, indicating that either $S$. sonne $i$ is more common in our environment or it is associated with more severe presentations, as patients with more severe disease are more likely to seek medical help. Indeed, most patients with shigellosis require immediate hospitalization [4]. Salmonella was also commonly found in our patients, but no typhoidal specimens were isolated. In our sample, E. coli was the least frequent causative agent of acute diarrhea; however, it is more important to the epidemiology of diarrhea in poorer areas $[10,11]$. No cases of Campylobacter-associated diarrhea were 
Table 3. Antimicrobial resistance of the bacterial specimens isolated from diarrheal stools

\begin{tabular}{lcclc}
\hline & Shigella spp. (141) & Salmonella sp. (100) & E. coli (19) & Total (260) \\
\hline $\begin{array}{l}\text { Ampicillin } \\
\text { sulbactam }\end{array}$ & $14(9.9 \%)$ & $5(5.0 \%)$ & $3(15.8 \%)$ & $22(8.5 \%)$ \\
Ampicillin & $31(22.0 \%)$ & $4(4.0 \%)$ & $5(26.3 \%)$ & $40(15.4 \%)$ \\
Aztreonan & - & - & - & - \\
Ceftazidime & - & $1(1.0 \%)$ & - & $1(0.4 \%)$ \\
Ceftriaxone & - & - & - & - \\
Ciprofloxacin & $1(0.7 \%)$ & $2(2.0 \%)$ & - & $3(1.2 \%)$ \\
Imipenen & $1(0.7 \%)$ & $1(1.0 \%)$ & $1(5.3 \%)$ & $3(1.2 \%)$ \\
Piperacillin & $12(8.5 \%)$ & $2(2.0 \%)$ & $4(21.0 \%)$ & $18(6.9 \%)$ \\
Trimethoprim + & $127(90.1 \%)$ & $2(2.0 \%)$ & $6(31.6 \%)$ & $135(51.9 \%)$ \\
sulfamethoxazole & & & & \\
\hline
\end{tabular}

detected. A low incidence of Campylobacter had already been reported in Brazil [12], while it is much more common in other countries [13-15].

Our data point to Salmonella as the most important causative agent of diarrhea in children younger than five years. Children younger than one year, however, are not expected to present Shigella-associated diarrhea, in contrast to a many children aged one to five years-old. These observations have important clinical implications, since Salmonella spp. were characteristically multi-susceptible, in contrast to Shigella spp. and E. coli (Table 3).

The highest levels of incidence were during the summer and the winter, when school vacations allow children to increase their exposure to environmental pathogens and to contaminated food. Consequently, we attribute the unexpected low incidence found in December to the habit of traveling out of town during this month.

In spite of the relatively small size of the sample, we believe that our data provide useful information about antimicrobial resistance. In order to help practitioners to choose an adequate antimicrobial drug to start empirical therapy in a patient with severe diarrhea without knowledge of a specific pathogen, we assessed the antimicrobial resistance patterns of all the pathogens that were isolated. We detected high resistance rates to TMP-SMX, ampicillin, ampicillin associated with sulbactam and piperacillin in Shigella spp. strains. Thus, we do not recommend any of these drugs for the empirical treatment of acute bloody diarrhea in children. In very severe cases, with evidence of dissemination of disease, intravenous ceftriaxone is the best choice for many, while other drugs can be adequate choices for the empirical treatment of severe acute diarrhea in children (Table 2). Obviously, as soon as the results of stool cultures are available, the therapy can be altered to a safer and/or cheaper drug based on the antimicrobial susceptibility pattern.

Separate assessment of antimicrobial resistance patterns indicates that Shigella is more difficult to treat and requires careful consideration at the choice of antimicrobial therapy. However, Shigella resistance has not reached the alarming multi-resistance rates reported in other countries, where resistance to ciprofloxacin and to ceftriaxone is a reality [16-18]. While we detected high resistance rates to TMP-SMX, ampicillin, ampicillin associated with sulbactam and piperacillin, no resistance to ceftriaxone was detected and only one out of $141(0.7 \%)$ Shigella isolates was resistant to ciprofloxacin. Shigella sonnei isolates presented the highest resistance rates to TMP-SMX: 94.7\%, 
compared to $71.4 \%$ for $S$. flexneri and $51.9 \%$ for all bacteria isolated. Shigella flexneri presented a high rate of resistance to ampicillin compared to $S$. sonnei (60.8\% and $12.4 \%$, respectively). We found that the Salmonella strains were multi-susceptible, as high rates of resistance were not found against any of the drugs. Interestingly, $2.0 \%$ of those strains were found to be resistant to ciprofloxacin. While the possibility of a false result should be considered because of the automated testing method, resistance to ciprofloxacin among nontyphoidal Salmonella strains isolated from stool specimens has already been reported from several parts of the world $[19,20]$. On the other hand, a multicenter Latin American study published by Gales et al. did not isolate any strain of Salmonella resistant to fluoroquinolones from blood samples [21]. However, Salmonella spp. strains isolated from blood samples are recognized to be more susceptible than those from stools [22]. Furthermore, the flouroquinolone tested by Gales et al. was gatifloxacin, a new 8-methoxifluroquinolone that is supposed to be active against most ciprofloxacin-resistant bacteria. E. coli isolates were found to have a high rate of resistance to piperacillin, ampicillin, ampicillin + sulbactam and TMPSMX. However, the interpretation of these data is clouded by the relatively few $E$. coli-associated cases (19, or $7.3 \%$ of the total). All in all, our susceptibility patterns are slightly more favorable than those reported by the large multicenter SENTRY Antimicrobial Surveillance Program Report for Brazil and Latin America [23].

Even though it is important to be careful with antimicrobial susceptibility patterns provided by automated methods, clinical practice has continuously endorsed their use. Furthermore, comparative studies have provided a very low testing discrepancy rate between the Walkaway system and the reference conventional agar dilution method for the identification and the antimicrobial susceptibility patterns of Shigella, Salmonella and E. coli [24-26]. However, no trial addressed any of those bacteria specifically nor have they involved a large number of isolates.

We did not assess the role of nalidixic acid in the treatment of acute diarrhea. In spite of the worldwide spread of resistant strains, the use of nalidixic acid is still recommended by the World Health Organization guidelines for the management of acute bloody diarrhea in children [27], and it may remain an important option for the treatment of acute infectious diarrhea, especially in services that cannot afford far more expensive drugs.

\section{References}

1. World Health Organization. Diarrhoeal disease due to Shigella disease. In: Vaccines, immunization and biologicals. Geneva: World Health Organization, 1998:1-5.

2. Shears P. Shigella infections. Ann Trop Med Parasitol 1996;90:105-14.

3. Tjaniadi P., Lesmana M., Subekti D., et al. Antimicrobial resistance of bacterial pathogens associated with diarrheal patients in Indonesia. Am J Trop Med Hyg 2003;68:666-70.

4. Niemogha M.T., Alabe S.A., Uzoma K.L., et al. The incidence of Salmonella, Shigella and other enteric bacterial pathogens in stool specimens of diarrhoea patients. Niger Med J 1995;28:70-4.

5. Wasfy M.O., Oyofo B.A., David J.C., et al. Isolation and antibiotic susceptibility of Salmonella, Shigella and Campylobacter from acute enteric infections in Egypt. J Health Popul Nutr 2000; $18: 33-8$.

6. Bennish M.L., Salam M.A., Hossain H.A., et al. Antimicrobial resistance to Shigella isolates in Bangladesh, 1983-1990: increasing frequency of strains multiply resistant to ampicillin, trimethoprimsulfamethoxazole and nalidixic acid. Clin Infect Dis 1992; $14: 1055-60$.

7. National Committee for Clinical Laboratory Standards. Performance standards for antimicrobial testing: sixth international supplement. Wayne PA: National Committee for Clinical Laboratory Standards, 1995 (NCCLS document no. MI00-S6).

8. Oldfield III E.C., Wallace M.R. The role of antibiotics in the treatment of infectious diarrhea. Gastroentrol Clin North Am 2001;30:817-36.

9. Goodman L., Segreti J. Infectious diarrhea. Dis Mon 1999;45:268-99.

10. Notario R., Borda N., Gambande T., Sutich E. Species and serovars of enteropathogenic agents associated with acute diarrheal disease in Rosario, Argentina. Rev Inst Med Trop Sao Paulo 1996;38:5-7.

11. Bojuwoye B.J. Pathogenic mechanisms of bacterial diarrhea: new insight and clinical implications. Nig Med Parasitol 1988;82:185-8. 
12. Toporovski M.S., Mimica I.M., Chieffi P.P., et al. Acute diarrhea in children less than 3 years of age: Enteropathogens isolated in patient's stools, compared with a control group [Article in Portuguese]. J Pediatr (Rio J) 1999;75:97-104.

13. Coker A.O., Isokpehi R.D., Thomas B.N., et al. Human campylobacteriosis in developing countries. Emerg Infect Dis 2002;8:237-44.

14. Ali A.M., Qureshi A.H., Rafi S., et al. Frequency of Campylobacter jejuni in diarrhoea/dysentery in children in Rawalpindi and Islamabad. J Pak Med Assoc 2003;53:517-20.

15. Isenbarger D.W., Hien B.T., Ha H.T., et al. Prospective study of the incidence of diarrhoea and prevalence of bacterial pathogens in a cohort of Vietnamese children along the Red River. Epidemiol Infect 2001;127:229-36.

16. Niyogi S.K., Mitra U., Dutta P. Changing pattern of serotypes and antimicrobial susceptibility of Shigella species isolated from children in Calcutta, India. Jpn J Infect Dis 2001;54:121-2.

17. Sur D., Niyogi S.K., Sur S., et al. Multidrug-resistant Shigella dysenteriae type 1: forerunners of a new epidemic strain in eastern India? Emerg Infect Dis 2003;9:404-5.

18. Gassama A., Sow P.S., Fall F., et al. Ordinary and opportunistic enteropathogens associated with diarrhea in Senegalese adults in relation to human immunodeficiency virus serostatus. Int $\mathbf{J}$ Infect Dis 2001;5:192-8.

19. Jiang Z.D., Lowe B., Verenkar M.P., et al. Prevalence of enteric pathogens among international travelers with diarrhea acquired in Kenya (Monbasa), India (Goa), or Jamaica (Montego Bay). J Infect Dis 2002;185:497-502.

20. Taneja N., Mohan B., Khurana S., Sharma M. Antimicrobial resistance in selected bacterial enteropathogens in north India. Indian J Med Res 2004;120:39-43.

21. Gales A.C., Sader H.S., Mendes R.E., Jones R.N. Salmonella spp. isolates causing bloodstream infections in Latin America: report of antimicrobial activity from the SENTRY Antimicrobial Surveillance Program (1997-2000). Diagn Microbiol Infect Dis 2002;44:313-8.

22. Oplustil C.P., Nunes R., Mendes C., RESISTNET Group. Multicenter evaluation of resistance patterns of Klebsiella pneumoniae, Escherichia coli, Salmonella spp. and Shigella spp. isolated from clinical specimens in Brazil: RESISTNET surveillance program. Braz J Infect Dis 2001;5:8-12.

23. Sader H.S., Jones R.N., Gales A.C., et al. SENTRY antimicrobial surveillance program report: Latin American and Brazilian results for 1997 through 2001. Braz J Infect Dis 2004;8(1):25-79.
24. Kelly M.T., Leicester C. Evaluation of the Autoscan Walkaway system for rapid identification and susceptibility testing of Gram-negative bacilli. J Clin Microbiol 1992;30(6):1568-71.

25. Odumeru J.A., Steele M., Fruhner L., et al. Evaluation of accuracy and repeatability of identification of foodborne pathogens by automated bacterial identification systems. J Clin Microbiol 1999;37(4):944-9.

26. McGregor A., Schio F., Beaton S., et al. The Microscan Walkaway diagnostic microbiology system - an evaluation. Pathology 1995;27(2):172-6.

27. World Health Organization. The management of bloody diarrhoea in young children. WHO, Geneva, Swizterland, 1994 (WHO/CDD/94.49). 